\title{
¿Se relacionan las creencias sobre las matemáticas con el rendimiento académico en matemáticas en estudiantes de contexto vulnerables?
}

\author{
Are mathematics beliefs related to academic performance in mathematics \\ in students from vulnerable contexts?
}

\author{
Gerson Adriano Rincón Alvareza, Raúl Prada Nuñez ${ }^{\mathrm{b}}$, Raquel. Fernández-Cézarc \\ ${ }^{a}$ Magister Neuropsicología y Educación, Proyecto Todos a Aprender (PTA), Secretaría de Educación Municipal Cúcuta, Ministerio de Educación \\ Nacional, GersonAdriano.Rincon@alu.uclm.es, 0000-0002-4375-1993, Cúcuta, Colombia \\ bMagister en educación matemática, Universidad Francisco de Paula Santander raul.prada@ufps.edu.co, 0000-0001-6145-1786, Cúcuta, Colombia. \\ 'Doctora en Ciencias Químicas, Universidad de Castilla La Mancha, raquel.fcezar@uclm.es, 0000-0002-9013-7734, Toledo, España
}

Forma de citar: Rincón-Alvarez. G.A, Prada-Nuñez. P, Fernández-Cézar. R . ¿Se relacionan las creencias sobre las matemáticas con el rendimiento académico en matemáticas en estudiantes de contexto vulnerables?. Eco Matemático, $10(2), 6-15$

Recibido: 3 febrero 2019

Aceptado: 5 abril 2019

\section{Palabras clave}

Educación matemática, Creencias, Dominio afectivo, Rendimiento académico.

\section{Keywords}

Mathematics Education, Beliefs, Affective domain,

\begin{abstract}
Resumen: El presente artículo es un avance de un estudio realizado para averiguar la relación existente entre el dominio afectivo en matemáticas (creencias, actitudes y emociones) y el rendimiento académico en esta área en estudiantes de contextos vulnerables. El objetivo es analizar las creencias de los estudiantes sobre las matemáticas y su posible relación con su rendimiento académico. La metodología empleada es de carácter cuantitativo, descriptivo y correlacional, con un diseño de tipo no experimental, transversal correlacional-causal, ya que busca explicar la relación entre las variables. La muestra estaba constituida por 121 estudiantes de 3er grado de educación básica primaria, perteneciente a una institución educativa pública de Cúcuta, Norte de Santander, Colombia, seleccionados mediante muestreo no probabilístico. El instrumento empleado para la recolección de datos fue un cuestionario estructurado con respuestas del tipo Escala de Likert, del cual se estudió su validez y confiabilidad. Para el rendimiento académico se utilizó el instrumento de caracterización de las habilidades y procedimientos que utilizan los estudiantes de 3er grado en el área de matemáticas, diseñado por el programa Todos a Aprender (PTA) del Ministerio de Educación Nacional (MEN). Los resultados evidencian que las creencias sobre las matemáticas, desde la perspectiva de los estudiantes, son un factor determinante del rendimiento académico en matemáticas de este tipo de alumnado.
\end{abstract}

*Autor para correspondencia GersonAdriano.Rincon@alu.uclm.es 
Academic performance.

\section{Introducción}

Cada vez es más frecuente que los docentes e investigadores de los procesos de enseñanza aprendizaje centren la atención en identificar los obstáculos y barreras que surgen para los estudiantes mientras aprenden. El bajo rendimiento académico, los altos índices de absentismo, las elevadas tasas de abandono, no solo en los niveles de educación media y superior, sino también en el de básica primaria, se han identificado como consecuencias de esos obstáculos que encuentran los alumnos, y se han constituido en preocupaciones de instituciones gubernamentales de educación, así como del profesorado de todos los niveles educativos y de la ciudadanía en general (Abalde, Barca, Muñoz, \& Ziemer, 2009). Dentro de este amplio campo, surge un gran interés en el estudio del dominio afectivo hacia las matemáticas, considerándolo compuesto por las creencias, actitudes y emociones en relación con la enseñanza/aprendizaje de las matemáticas (Ramírez, Prada, Hernández, \& Fernández, 2018). Es un hecho que la compleja sociedad actual exige aprender matemáticas para desenvolverse adecuadamente en ella, y manejar y entender los avances tecnológicos. También la progresiva importancia que van adquiriendo los medios de comunicación obligan a la adaptación de las personas a nuevas formas de socializar a raíz del cambio social (Ignacio, Barona \& Nieto, 2006) actualmente producido. Pero también se evidencia que, a pesar de su importancia y utilidad, las matemáticas suelen ser vistas y valoradas por la mayor parte de las personas, y en especial de los estudiantes, como una materia sin utilidad en la vida, difícil, aburrida, abstracta, en general poco práctica, percibiendo que su aprendizaje requiere de un alto nivel de dedicación, concentración e inteligencia que no está al alcance de todos (Araya, 2014). Estas creencias generalizadas sobre las matemáticas muy posiblemente influyen en el abandono de la misma, e incluso pueden ser causantes de la deserción escolar (Jerez, 2014).

Teniendo presente lo anterior, se requiere de un cambio en la percepción del área de matemáticas, por parte de los estudiantes y posiblemente también de los docentes que la orientan, en especial en la básica primaria en Colombia. Este profesorado, por lo general tiende, a ser de otras especialidades, aunque, por necesidad del servicio, son encargados de enseñar esta materia por los equipos directivos de los centros. En este contexto, el presente trabajo fue desarrollado para determinar en qué medida existe relación entre el dominio afectivo hacia las matemáticas y el rendimiento académico del estudiante en entornos sociales vulnerables.

En los últimos años el Ministerio de Educación Nacional (MEN), a través del Proyecto Todos a Aprender (PTA), está realizando una restructuración curricular en el área de matemáticas de la etapa básica primaria, apoyándose en los referentes de calidad recogidos en la Ley General de educación (MinEducacion, 1994), Lineamientos curriculares (MinEducacion, 1998), Estándares Básicos de Competencias (MinEducacion, 2003), los Derechos Básicos de Aprendizaje (MinEducacion, 2016), y las mallas de aprendizaje (MinEducacion, 2017). 
Es claro que todos estos referentes de calidad buscan mejorar el rendimiento académico en general, y el de matemáticas en particular, de la ciudadanía colombiana. Por ello es importante tener claro este concepto desde la perspectiva del desarrollo humano y planteándose al estudiante como constructor de su propio aprendizaje. A este respecto, se considera el rendimiento académico como un fenómeno educativo de carácter complejo e integral, que involucra tanto a los estudiantes, con sus logros y experiencias en la educación básica, como a las diversas dimensiones institucionales que contribuyen a la formación integral de este (Zuluaga, Rengifo \& López, 2012).

Por ello la conceptualización del rendimiento académico es compleja, utilizándose diferentes denominaciones como: aptitud escolar, desempeño académico o rendimiento escolar. Pero generalmente las diferencias de concepto son solo semánticas, como recoge Edel (2003). Este autor expresa que una de las variables más empleadas por los docentes e investigadores para al rendimiento académico son las calificaciones. Por esta razón se encuentran estudios que pretenden calcular algunos índices de fiabilidad y validez de este criterio considerado como 'predictivo' del rendimiento académico. las calificaciones son reflejo de las evaluaciones y/o exámenes donde el estudiante ha de demostrar sus conocimientos sobre las distintas áreas o materias (Cascón, 2000).

Según Edel (2003), el rendimiento académico es un constructo que puede medirse cuantitativa o cualitativamente, recabando evidencias de las habilidades, conocimientos, actitudes y valores desarrollados por el estudiante en el proceso de enseñanza aprendizaje. Además, en el rendimiento académico intervienen varios factores, como aspectos personales (cognitivos, biológicos y socioafectivos) del estudiante, y aspectos contextuales (sociales y culturales), según los autores Barrios y Frías (2016). Por otro lado, para Martí (2003), rendimiento académico insatisfactorio en un estudiante, es aquel que se sitúa por debajo del rendimiento esperado por él o por sus mayores, es decir, aquel que no cumple las expectativas. Por ello se evidencia la importancia de establecer el análisis del rendimiento más allá de las calificaciones, considerando todas sus dimensiones, de manera que facilite una posible explicación para el fracaso y el abandono escolares, y pueda contribuirse a fortalecer la permanencia en el sistema educativo.

Esas dimensiones que son reconocidas como incidentales en el rendimiento académico de los estudiantes, son aquellas que se asocian directamente con la estructura de la sociedad no solo colombiana si no latino americana. La cultura, el ordenamiento político y la organización socioeconómica son determinantes en el desempeño del estudiante, transformándose en un importante grupo de variables que deben ser consideradas al momento de pensar en proyectos que estudien el rendimiento académico y que conlleve a una posible desigualad de la educación (Cornejo y Redondo, 2001). Lo que sucede con estas variables es que requieren de Políticas Públicas a largo plazo un trabajo y de trabajo constante para poder modificar sus efectos en lo cual Colombia está apostando a través del programa Todos a aprender (PTA).

Estando muy relacionado al anterior grupo de dimensiones o factores también existen los que son caracterizados como elementos provenientes del entorno sociocultural cercano del estudiante y que impactan en la comunidad educativa modificando la labor en la escuela de docentes y estudiantes. Los orígenes socioculturales de la familia, los capitales culturales de los padres, el nivel socioeconómico, entre otros, pasan a jugar parte importante en esta compleja combinación de factores que afectan el desempeño de los estudiantes (Bourdieu, 2003; Duarte, 2002).

Teniendo presente los factores expuestos en los párrafos anteriores e identificando las características de la institución objeto de estudio que se 
encuentra ubica en un barrio periférico de la ciudad de Cúcuta formado por familias desplazadas de las zonas rurales por el conflicto armado que vive el país y aunado a esta población en los últimos años las familias migrantes venezolanas ya que la ciudad es frontera con este país, la comunidad educativa que conforma esta institución son personas que se encuentran en un contexto de vulnerabilidad. Aclarando que el término "vulnerabilidad" refiere a la probabilidad de daño físico y moral. En el ámbito social, Castel (2004) alude a este concepto como una zona de precarización del empleo, pobreza socioeconómica, fracaso escolar, precarios soportes sociales en salud, en prevención y ambientales de la vida social que se encuentra interceptando la integración (zona social de trabajo y soporte social firme) y la exclusión (zona social de desafiliación social, discriminación y estigmatización). La exclusión social, o desafiliación en términos de Castel, apunta a un proceso histórico de degradación de las relaciones de trabajo y de los soportes de integración social.

De acuerdo con lo anterior, para este estudio piloto, en el contraste de hipótesis se plantearon las siguientes:

$\mathrm{H}_{1}$ : Existe relación entre las creencias sobre las matemáticas y el rendimiento académico de los estudiantes.

$\mathrm{H}_{0}$ : No existe relación entre las creencias sobre las matemáticas y el rendimiento académico de los estudiantes.

\section{Metodología}

El enfoque de la investigación es cuantitativo. En cuanto al diseño es de tipo no experimental, transversal correlacional-causal (Arias, 2012), pues permite determinar las relaciones entre las creencias sobre las matemáticas y el rendimiento académico.

\section{Población y Muestra}

La población estuvo conformada por 562 estudiantes de género masculino y femenino de la básica primaria de la Institución Educativa Mariano Ospina Rodríguez sede Monseñor Luis Pérez Hernández municipio de Cúcuta, Norte de Santander. La muestra quedo conformada por 121 estudiantes de grado tercero de primaria con edades comprendidas entre los 8 a 11 años, matriculados en el año 2019. Los participantes aceptaron formar parte del estudio voluntariamente en el estudio, para lo que se recabó el consentimiento informado de sus padres, esta muestra fue tomada en forma no probabilística por conveniencia.

\section{Instrumentos}

La técnica utilizada para la obtención de los datos del dominio afectivo en la presente investigación fue un cuestionario. El instrumento utilizado fue un cuestionario dividido en 3 secciones: la primera de información general, la segunda del dominio afectivo hacia las matemáticas (13 ítems asociados con las Creencias, 15 ítems asociados con las Actitudes, y 10 ítems asociados con las Emociones), y la tercera sección incluía los ítems sobre Procesos Matemáticos en la Práctica Docente. La consistencia interna valoró mediante la prueba Alfa de Cronbach, obteniendo el resultado de .713.

En este trabajo se presentan las siguientes variables:

Creencias sobre las matemáticas. Consta de un cuestionario estilo escala Likert de 13 preguntas. Los estudiantes respondieron con respecto al cuestionario eligiendo entre 1, que significa "Estoy muy en desacuerdo", 2, "Estoy en desacuerdo", 3, "No estoy en desacuerdo ni estoy de acuerdo", 4, "Estoy de acuerdo", 5, "Estoy muy de acuerdo", según fuese su nivel de aprobación. 


\begin{tabular}{|c|c|c|c|c|c|}
\hline \multicolumn{6}{|l|}{ Segunda Sección. Dominio Afectivo hacia las Matemáticas } \\
\hline Ítems asociados con las Creencias sobre las Matemáticas & 1 & 2 & 3 & 4 & 5 \\
\hline$\checkmark \quad$ Las matemáticas son útiles y necesarias en todos los aspectos de la vida. & & & & & \\
\hline$\checkmark$ Las matemáticas son dificiles, aburridas y alejadas de la realidad. & & & & & \\
\hline$\checkmark$ En matemáticas es fundamental aprenderse de memoria los conceptos, fórmulas y reglas. & & & & & \\
\hline $\begin{array}{l}\checkmark \text { Los ejercicios de matemáticas se resuelven rápidamente si se conoce la fórmula, regla } 0 \\
\text { procedimiento. }\end{array}$ & & & & & \\
\hline$\checkmark \quad$ Para aprender matemáticas debo estudiar por mi cuenta. & & & & & \\
\hline $\begin{array}{l}\text { Cuando resuelvo un ejercicio matemático, me importa más el resultado que el proceso } \\
\text { utilizado. }\end{array}$ & & & & & \\
\hline $\begin{array}{l}\text { La forma de resolver ejercicios matemáticos en clase, es diferente a la que se utiliza para } \\
\text { resolver situaciones de la vida cotidiana en dónde se requiera de las matemáticas. }\end{array}$ & & & & & \\
\hline$\checkmark \quad$ Busco distintas maneras y formas para resolver ejercicios en Matemáticas. & & & & & \\
\hline$\checkmark$ A partir de los realizado en clase, puedo inventar mis propios ejercicios de matemáticas. & & & & & \\
\hline$\checkmark$ Al entender las matemáticas, me ayuda a resolver dudas de otras asignaturas. & & & & & \\
\hline$\checkmark$ Cuando resuelvo un ejercicio en matemáticas, me siento seguro de la respuesta es correcta. & & & & & \\
\hline$\checkmark$ Me considero muy capaz y hábil en matemáticas. & & & & & \\
\hline Las matemáticas son para personas inteligentes y creativas. & & & & & \\
\hline
\end{tabular}

Figura 1. Ítems asociados con las Creencias sobre las Matemáticas

Rendimiento Académico. En esta variable se consideró el instrumento de caracterización de las habilidades y procedimientos que utilizan los estudiantes de tercer grado en el área de matemáticas, diseñado por el programa Todos a Aprender (PTA) del MEN. Contiene 8 tareas, cada tarea conformada por 4 ejercicios asociados a las habilidades básicas, las cuales a su vez están relacionadas con los Derechos Básicos de Aprendizaje (Min Educación, 2016) y los ejes de progresión de los pensamientos numérico y variacional (Min Educación, 2017). Rendimiento Académico. En esta variable se consideró el instrumento de caracterización de las habilidades y procedimientos que utilizan los estudiantes de tercer grado en el área de matemáticas, diseñado por el programa Todos a Aprender (PTA) del MEN. Contiene 8 tareas, cada tarea conformada por 4 ejercicios asociados a las habilidades básicas, las cuales a su vez están relacionadas con los Derechos Básicos de Aprendizaje (Min Educación, 2016) y los ejes de progresión de los pensamientos numérico y variacional (Min Educación, 2017). 

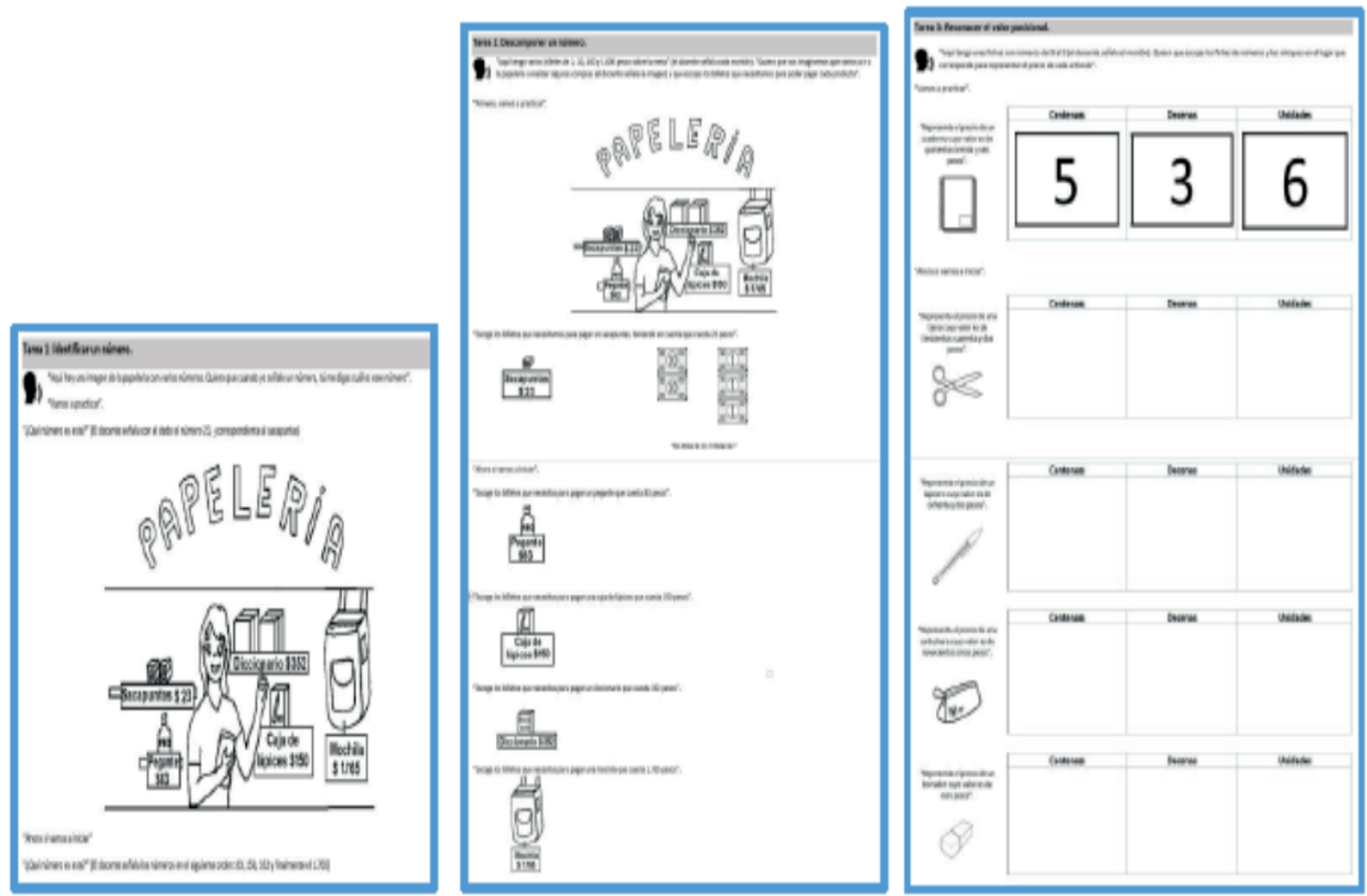

Figura 2. Primer momento instrumento de caracterización de las habilidades y procedimientos que utilizan los estudiantes de tercer grado en el área de matemáticas
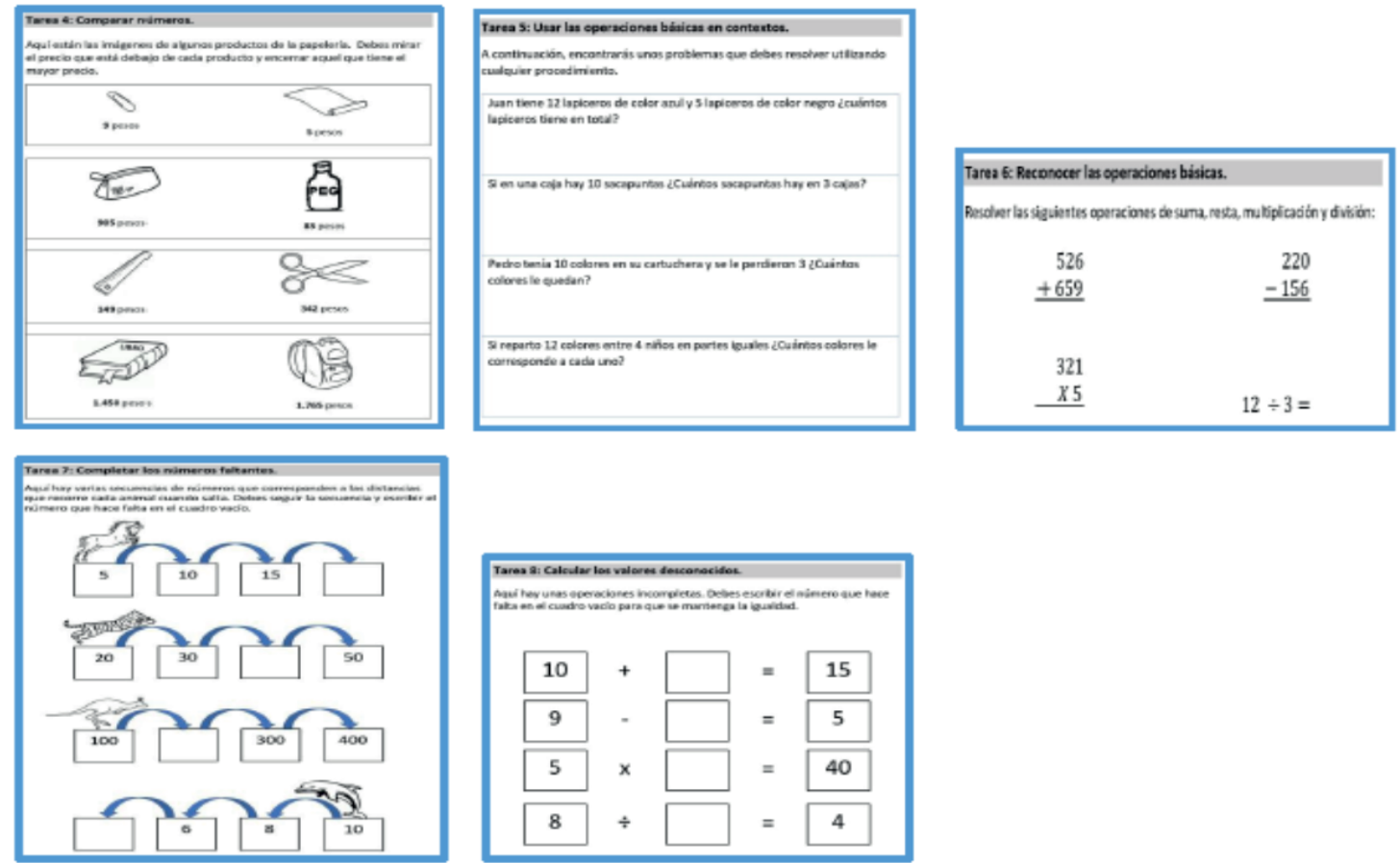

Figura 3. Segundo momento instrumento de caracterización de las habilidades y procedimientos que utilizan los estudiantes de tercer grado en el área de matemáticas 


\section{Procedimiento.}

Se solicitó el permiso y autorización en forma escrita a la Rectoría de la Institución Educativa. La obtención de los datos se realizó durante el desarrollo de las clases, en coordinación con el docente de aula. Antes de que los estudiantes respondieran al cuestionario se dio las indicaciones pertinentes. Se contó con la colaboración de un asistente en la aplicación del cuestionario.

El instrumento de Rendimiento académico se aplicó en dos momentos. En el primero se entrevistó a los sujetos en un contexto ambientado en una tienda escolar. Se les pasaron las tareas 1-3. En el segundo momento se pasaron de manera individual las tareas 4, 5, 6, 7 y 8, que implicaban preguntas de elección múltiple con una única respuesta correcta. En ambos casos los participantes dispusieron de material concreto para facilitar las operaciones.

\section{Procesamiento y análisis de la información}

Para la presentación de los resultados se utilizó la hoja de cálculo Excel en la edición de tablas y figuras, así como el software SPSS, versión 22 para los contrastes de hipótesis estadísticas y cálculo de correlaciones. Para conocer el grado de asociación (coeficiente de correlación) entre las variables de estudio, se utilizó el Rho de Spearman con el cual se puede determinar la dependencia o independencia de dos variables aleatorias (Pérez-Tejada, 1998). El coeficiente de correlación Rho de Spearman es el coeficiente usado cuando las variables son ordinales, o se incumple el supuesto de normalidad (aproximación no paramétrica)

\section{Resultados}

La percepción de los estudiantes con relación a las creencias sobre las matemáticas en la muestra estudiada se expone en la Tabla I.
Tabla I. Percepción del estudiante, creencias sobre las matemáticas

\begin{tabular}{ccc}
\hline Nivel de creencias sobre las matemáticas & \# de estudiantes & Porcentaje \\
& & \\
\hline Totalmente en desacuerdo & 5 & $4.1 \%$ \\
Desacuerdo & 22 & $18.2 \%$ \\
Ni rechaza ni acepta & 45 & $37.2 \%$ \\
De acuerdo & 31 & $25.6 \%$ \\
Totalmente de acuerdo & 18 & $14.9 \%$ \\
Total & 121 & $100 \%$ \\
\hline
\end{tabular}

El promedio de ejercicios desarrollado correctamente por tarea en relación con su rendimiento académico de la muestra estudiada se expone en la Tabla II.

Tabla II. Pregunta promedio por tarea contestada correctamente por los estudiantes

\begin{tabular}{lcc}
\hline \multicolumn{1}{c}{ Preguntas promedio por tareas } & \#de estudiantes & Porcentaje \\
\hline 1 (nivel bajo) & 2 & $1.7 \%$ \\
2 (nivel medio) & 19 & $15.7 \%$ \\
3 (nivel alto) & 72 & $59.5 \%$ \\
4 (nivel superior) & 28 & $23.1 \%$ \\
Total & 121 & $100,0 \%$
\end{tabular}

La Tabla III muestra la correlación Rho de Spearman entre las variables creencias sobre matemáticas y el rendimiento académico.

Tabla III. Relación entre el nivel del rendimiento académico de los estudiantes y la práctica pedagógica en los procesos matemáticos de los docentes

\begin{tabular}{|c|c|c|c|c|c|}
\hline & \multicolumn{5}{|c|}{ Conrelaciones } \\
\hline & & & Rendimiento académico & $\begin{array}{l}\text { Creencias sobre } \\
\text { matematicas }\end{array}$ & las \\
\hline \multirow{6}{*}{$\begin{array}{l}\text { Rho de } \\
\text { Spearman }\end{array}$} & Rendimiento Académico & Coeficiente de comelación & 1,000 & 0.883 & \\
\hline & & Sig. (bilateral) & & 0.000 & \\
\hline & & $\mathrm{N}$ & 121 & 121 & \\
\hline & Creencias sobre las & Coeficiente de correlación & 0.883 & 1.000 & \\
\hline & matemáticas & Sig. (bilateral) & 0.000 & . & \\
\hline & & $\mathrm{N}$ & 121 & 121 & \\
\hline
\end{tabular}

\section{Discusión}

En este estudio piloto se validó la hipótesis de investigación que fue confirmada a partir de la información obtenida de la muestra exponiendo que existe una relación significativa entre las creencias en matemáticas y el rendimiento académico de los estudiantes de tercer grado de educación básica 
primaria de la institución educativa objeto de estudio.

Entre los resultados se encuentra que las creencias del estudiantado son mayoritariamente neutras, pues el $37.2 \%$ no tiene una creencia ni positiva ni negativa hacia las matemáticas, seguido que un $25.6 \%$ que están de acuerdo, concordando con Vázquez, Bellón y Fernández (2014).

Por otro lado, al respecto del rendimiento, la gran mayoría de los estudiantes, $82.6 \%$, contestaron bien en promedio 3 o los 4 ejercicios que se tenía por cada una de las 8 tareas encontrándose en los niveles altos o superior. Podrían deberse este alto rendimiento a la influencia de las prácticas pedagógicas y el dominio de los contenidos de matemática por parte del docente (Caciá, Reyes, Rosales y Marroquín, 2012). Ello mediaría una mejor comprensión y aprendizaje de los temas de la matemática por parte de los estudiantes, como afirma López-Quijano (2014). Por otro lado, los autores como Lester, Garofalo y Kroll (1989), señalan que las creencias de los alumnos acerca de la resolución de los problemas tienden a afectar a su autoconfianza en esa actividad, aumentándola o disminuyéndola. En este caso, al parecer, las creencias positivas hacia las matemáticas favorecen su autoconfianza en el desempeño de estas.

La correlación entre estas dos variables, creencias y rendimiento se observa que es positiva es decir al aumentar la positividad de las creencias sobre matemáticas en los estudiantes, aumenta el rendimiento académico de estos, y viceversa. Se obtiene una fuerte correlación, pues el valor de $r$ $=.883$, cercano a $1, \mathrm{y}$ un $\mathrm{p}$-valor estadísticamente significativo $(\mathrm{p}<.01)$. Podemos, por tanto, afirmar que se rechaza la hipótesis nula $(\mathrm{H} 0 ; \mathrm{p}<.01)$, resultando evidencia estadística suficiente para aceptar que existe relación entre las creencias sobre matemáticas y rendimiento académico en los estudiantes participantes en el estudio. Estos resultados están en línea con los obtenidos por
Vázquez, Bellón y Fernández (2014), y Bazán y Aparicio (2006), que correlación positiva y significativa entre creencias y rendimiento, de forma que aumentan las calificaciones en función de las actitudes, entre las que sitúan a las creencias positivas hacia las matemáticas. Sin embargo, en su caso el alumnado no presentaba especial vulnerabilidad, como es el caso de los participantes en este trabajo.

Se encuentran también otros estudios alineados con el presente en cuanto a la correlación fuerte y positiva entre creencias y rendimiento del estudiantado de básica primaria. Estos son el estudio de Pintrich, Anderman y Klobucar (1994), y el Navas, Sampascual y Castejón (1994). En estos trabajos se pone de manifiesto el logro del éxito a la relación de causas internas o intrapersonales controlables como el esfuerzo, la capacidad, la dedicación con los aspectos motivacionales y cognitivos. Los resultados de estos estudios han confirmado que aquellos estudiantes que atribuyeron el éxito a causas internas y al control se mostraron menos ansiosos, con más expectativas de éxito, más orientados al dominio, autoeficaces y metacognitivos y, además, rindieron mejor. Adicionalmente, los estudiantes que creían que su fracaso era debido a factores inestables e incontrolables eran menos proclives al dominio de la situación, eran menos eficaces, tenían menores expectativas de logro y más bajo rendimiento académico. Sin embargo en el trabajo presente no se ha indagado entre esas posibles causas de la correlación positiva entre rendimiento y creencias, lo que quedaría para una siguiente etapa de la investigación.

\section{Conclusiones}

Se analizaron los resultados parciales (avance de investigación) de la relación entre creencias sobre las matemáticas y el rendimiento académico en una institución de educación básica primaria, específicamente en tercer grado. Como corresponde a un estudio piloto, el objeto del mismo no ha sido generalizar esta relación sino tener una aproximación 
a la situación para así poder determinar con más precisión la relación entre estas variables en un estudio posterior que incluya un número mayor de estudiantes de todos los grados de diferentes instituciones educativas de nivel primaria secundaria técnica y universitaria del Departamento Norte de Santander - Colombia. De esta manera, se podrá establecer una triangulación entre los antecedentes y las posturas teóricas que lo sustentan, además de dar cuenta de las relaciones explicativas del rendimiento académico relativas a las creencias, actitudes y emociones como constructos del dominio afectivo de los estudiantes y la práctica pedagógica que incluye a los procesos matemáticos de los docentes.

En particular con respecto a la muestra de estudio se evidencia una relación positiva entre las creencias sobre las matemáticas entre los estudiantes de tercer grado de primaria y su rendimiento académico, indicando que a mayor percepción positiva en las creencias mayor es su rendimiento académico o que a menor percepción menor es su rendimiento académico.

\section{Referencias}

Abalde Paz, E., Barca Lozano, A., Muñoz Cantero, J. M., \& Ziemer, M. F. (2009). Rendimiento académico y enfoques de Aprendizaje: una aproximación a la realidad de la enseñanza superior brasileña en la región norte. Revista de Investigación Educativa, 27(2), 303-319.

Aguayo, M., \& Lora, E. (2007). Como realizar paso a paso un contraste de hipótesis con SPSS para Windows:(III) Relación o asociación del análisis de la dependencia (o no) entre dos variables cuantitativas. Correlación y regresión lineal simple. Disponible en http://www.fabis.org/ $\mathrm{html} /$ archivos/docuweb/contraste_hipotesis_3r. pdf

Araya, R. G. (2014). Relación entre la dimensión afectiva y el aprendizaje de las matemáticas. Revista Electrónica Educare, 18(2), 117-139.

Arias, F. (2012). El proyecto de investigación. Introducción a la metodología de investigación científica. Caracas: Editorial Episteme.

Barrios, M. \& Frías, M. (2016). Factores que influyen en el desarrollo y rendimiento escolar de los jóvenes de bachillerato. Revista Colombiana de Psicología, 25(1), 63-82. DOI: https://doi. org/10.15446/rcp.v25n1.46921

Bazán, J. L., \& Aparicio A. S. (2006). Las actitudes hacia la Matemática-Estadística dentro de un modelo de aprendizaje. Revista Semestral del Departamento de Educación, 25(28), 1-12.

Bourdieu, P. y Passeron, J. C. (2003): Los Herederos. Buenos Aires: Ediciones Siglo XXI

Caciá, D., Reyes, M., Rosales, C. y Marroquín, E. (2012). Factores que inciden en el rendimiento en matemática de niñas y niños del primer ciclo del nivel de educación primaria en escuelas públicas de Guatemala. Guatemala: Ministerio de Educación de Guatemala.

Cascón, I. (2000). Análisis de las calificaciones escolares como criterio de rendimiento académico. Recuperado de https://campus. usal.es/ inico/investigacion/jornadas/jornada2/ comun/c17.html

Castel, R. (2004). "Encuadre de la exclusión", en C. S. Karsz, La exclusión: bordeando susfronteras, Barcelona: Gedisa, pp. 55-86.

Colombia, C. D. (1994). Ley 115 de febrero 8 de 1994. Ley general de educación.

Cornejo, R. y Redondo, J. M. (2001): "El clima escolar percibido por los alumnos de enseñanza media”. Revista Última Década, n. ${ }^{\circ} 15$, Viña del Mar.

Duarte, K. (2002): "Mundos jóvenes, mundos adultos: Lo generacional y la reconstrucción de los puentes rotos en el liceo". Revista Última Década, n. ${ }^{\circ} 16$, Viña del Mar

Edel, R. (2003). El rendimiento académico: concepto, investigación y desarrollo. REICE. Revista Iberoamericana sobre Calidad, Eficacia y Cambio en Educación, 1(2), 1-16. Recuperado de https://revistas.uam.es/index.php/reice/ article/view/5354

Ignacio, N. G., Barona, E. G., \& Nieto, L. B. (2006). El dominio afectivo en el aprendizaje de las 
Matemáticas. Electronic Journal of Research in Educational Psychology, 4(1), 47-72.

Jerez Rondón, J. C. (2014). Creencias sobre la matemática en estudiantes de primer año de educación media de la UEB "Maporal". Municipio Rojas, Estado Barinas (Master's thesis).

Lester, F., Garofalo, J. y Kroll, D. (1989). SelfConfiance, Interest, Beliefs and Metacognition: Key Influences on Problem Solving Behavior. En D.B. McLeod y V. Adams (Eds.), Affect and Mahematical Problem Solving: A New Perspective (pp. 68-75). New York: SpringerVerlag.

López-Quijano, G. (2014). La enseñanza de las matemáticas, un reto para los maestros del siglo XXI. Praxis Pedagógica, 14(15), 5576. DOI https://doi.org/10.26620/uniminuto. praxis.14.15.2014.55-76

Martí, E. (2003). Representar el mundo externamente. La construcción infantil de los sistemas externos de representación. Madrid: A. Machado.

Ministerio de Educación Nacional (1998). Serie lineamientos curriculares: Matemáticas. Bogotá, Colombia: Min Educación.

Ministerio de Educación Nacional. (2003). Estándares básicos de competencias en matemáticas. Potenciar el pensamiento matemático: ¡un reto escolar! Bogotá, Colombia: MinEducación

Ministerio de Educación Nacional. (2016). Derechos básicos de aprendizaje Matemáticas V2. Bogotá, Colombia: Min Educación. Recuperado de http:// aprende.colombiaaprende.edu.co/sites/default/ files/naspublic/DBA_Matem\%C3\%A1ticas.pdf

Ministerio de Educación Nacional. (2017). Mallas de aprendizaje y DBA. Bogotá, Colombia: MinEducación. Recuperado de http://aprende. colombiaaprende.edu.co/es/node/89839

Pérez-Tejada, H. (1998). Estadística para las ciencias sociales, del comportamiento y de la salud. México, D.F.: Cengage Learning.

Pintrich, P.R., Anderman, E.M. y Klobucar, C H.
(1994). Intraindividual differences in motivation and cognition in students with and without learning disabilities. Journal of Learning Disabilities, 27, 360-370.

Ramírez Leal, Prada Núñez, R., Hernández Suárez, C. A., \& Fernández Cézar, R. (2018). Dominio afectivo y prácticas pedagógicas de docentes de Matemáticas. Revista Espacios, 39(23), 25-35

Sampascual, G., Navas, L. y Castejón, J.L. (1994). Procesos atribucionales en la Educación Secundaria Obligatoria: un análisis para la reflexión. Revista de Psicología General y Aplicada, 47, 449-459

Vázquez, M. D. M., Bellón, E. E., \& Fernández, R. C. (2014). Dimensión afectiva hacia la matemática: resultados de un análisis en educación primaria. Revista de Investigación Educativa, 32(1), 5772.

Zuluaga, Y. E. L., Rengifo, J. C. R., \& López, H. H. V. (2012). La práctica pedagógica de los docentes universitarios en el área de la salud y su relación con el desempeño académico. Revista Latinoamericana de Estudios Educativos (Colombia), 8(1), 95-118. 